\title{
COMPARISON OF R WAVE AMPLITUDE CHANGES VERSUS ST-SEGMENT DEPRESSION IN STRESS TESTING ELECTROCARDIOGRAM AMONG ISCHEMIC HEART DISEASE PATIENTS
}

By

\author{
Muhamad Abd Al-Hamied Muhamad Abou-Zeid, Ahmed M. Kamal \\ Motawh and Attia Morsy Shokr
}

Department of Cardiovascular Medicine, Faculty of Medicine, Al-Azhar University, Egypt

*Corresponding author: Muhamad Abd Al-Hamied Abou-Zeid,

E-mail: muhamadabouzeid87@gmail.com

\begin{abstract}
Background: R-wave changes might be helpful in accurate diagnosis of coronary artery disease with exercise testing in combination with ST segment changes as $\triangle \mathrm{RST}$ index and might be superior to the traditional ST segment depression only.

Objective: To further clarify R-wave amplitude (RWA) changes during exercise stress test (EST) among established IHD patients.

Patients and methods: The study enrolled 50 subjects - aged from above 35 to below 65 years old of both sexes, known to be ischemic by a previous angiography, divided into two main groups: Group I (control group): 15 subjects with normal coronaries (lesion < $30 \%$ stenosis), and Group II (IHD group): 35 patients with stable ischemic heart disease which were subdivided according to previous coronary angiography results (lesion $>70 \%$ stenosis). The study was conducted at the Department of Cardiovascular Medicine and its Outpatient Clinic, Al-Hussein University Hospital during the period from September 2019 to March 2021.

$\triangle \mathrm{RST}$ index was formed from algebraic summation of RWA difference between (rest and immediate recovery) plus ST segment depression in mile meters.

Results: $\triangle$ RST index was more useful in detection of coronary artery disease with higher sensitivity and specify among chronic ischemic patients than RWA difference or ST segment depression alone. When cutoff $\geq-1.65$ cardiac ischemia could be predicted with a sensitivity rate of $98.93 \%$, a specificity rate of $96.00 \%$, a positive predictive value of $96.3 \%$, and a negative predictive value of $98 \%$. RWA at a cut-off point of -1.2 was sensitive $95.02 \%$ and specific $94.0 \%$, a positive predictive value of $95.7 \%$, and a negative predictive value of $96.6 \%$. ST segment depression at a cut-off point of $\geq 0.45$ was sensitive $77.1 \%$ and specific $80 \%$, a positive predictive value of $78.4 \%$, and a negative predictive value of $60.3 \%$.
\end{abstract}

Conclusion: $\triangle \mathrm{RST}$ index was more useful in detection of coronary artery disease with higher sensitivity and specify among chronic ischemic patients than RWA difference or ST segment depression alone.

Keywords: Ischemic heart disease, R-wave amplitude, Exercise stress test, ST-Segment.

\section{INTRODUCTION}

Coronary artery disease (CAD) is considered a leading cause of morbidity and mortality in the world, and its incidence has been gradually increasing. CAD is a progressive inflammatory disease in which atherosclerosis plays a major role in its etiology (Mehta et al., 2015). 
Atherosclerosis progresses, especially if left untreated, symptoms may occur. They are most likely to occur during exercise or emotional stress, when the demand for the oxygen carried by the blood increases (O'Gara et al., 2013).

The exercise stress test (EST) is performed routinely in cardiology and commonly used for evaluation of prognosis and as a gateway to other imaging modalities. Stand-alone testing for CAD diagnosis is reserved for patients with intermediate risk for CAD and should be ordered with a careful understanding of the limitations of the test for this purpose. The advantages of exercise electrocardiographic testing are its ability to assess a variety of prognostic markers, most importantly functional capacity, which is a powerful predictor of mortality, widespread availability, safety, ease of administration, and relatively low cost. EST is conducted while monitoring the dynamic electrocardiogram changes (ECG), ventilatory threshold, blood pressure (BP), heart rate (HR), oxygen consumption, general patient self-reported effort (e.g., by a Borg Scale test) and physical appearance-including chest pain and dyspnea (Greenslade et al., 2015).

Exercise stress testing can be accomplished by either treadmill or cycle ergometer, although treadmills are predominantly used. There are several standard treadmill protocols that can be customized for patients to reach the endpoint of $85 \%$ to $100 \%$ maximum predicted heart rate. Among them, the Bruce protocol and modified Bruce are most popular. These protocols consist of 3-minute stages with incremental increases in speed and incline (Macintyre et al., 2013).

Horizontal or down sloping ST depression greater than or equal to $1 \mathrm{~mm}$ for at least $60-80 \mathrm{~ms}$ is commonly considered a positive stress test. However, the capacity of a stress test to localize ischemia based on leads demonstrating ischemic changes is poor. Patients with equivocal (up sloping) or non-diagnostic stress test results may be recommended for radionuclide myocardial perfusion image (MPI) or echocardiographic stress testing before a final decision regarding coronary angiography is made (Skyler et al., 2017).

Many factors affect the R-wave amplitude response to exercise. R-wave amplitude typically increases from rest to submaximal exercise, then decreases to a minimum at maximal exercise. If a patient were limited by objective signs or subjective symptoms, R-wave amplitude would increase from rest to such an end point. Such patients may be demonstrating a normal R-wave response but are classified as abnormal because of a submaximal effort. Exercise induced changes in R-wave amplitude have no independent predictive power but are associated with CAD because such patients are often sub maximally tested, and an R-wave decrease normally occurs at maximal exercise (Mittleman et al., 2011).

The aim of the present study was to clarify R-wave amplitude (RWA) changes during exercise stress test (EST) among established IHD patients. 


\section{PATIENTS AND METHODS}

This prospective cohort study on cases conducted at the Department of Cardiovascular Medicine and its Outpatient Clinic, Al-Hussein University Hospital during the period from September 2019 to March 2021. The study enrolled 50 subjects aged from above 35 to below 65 years old of both sexes, known to be ischemic on grounds of previous angiography, divided into two main groups as follows:

Group I (control group): Fifteen subjects with normal coronaries according to coronary angiography (lesion $<30 \%$ stenosis).

Group II (IHD group): Thirty five patients with stable ischemic heart disease which are subdivided according to previous coronary angiography results (lesion $>70 \%$ stenosis).

Inclusion Criteria: Controlled blood sugar, blood pressure, normal hepatic, renal functions, and previously documented ischemic heart patients on grounds of angiography.

Exclusion Criteria: Patients ages $>65 y \mathrm{ys}$ $\&$ <35ys, other causes of coronary ischemia such as congenital coronaries malformations, recent acute MI (within $2 \mathrm{ds}$ ), and valvular heart diseases.

\section{All the patients underwent detailed clinical evaluation that included:}

1. Complete history taking: Full history was taken from the patients regarding the age, sex and risk factors of CAD including: smoking, hypertension, diabetes mellitus, dyslipidemia and family history of atherosclerosis.
2. General and cardiac examination: Vital signs (blood pressure, pulse, chest examination), and heart examination (normal heart sounds, abnormal heart sounds).

\section{Resting 12-lead ECG by Philips TC20 electrocardiograph machine:}

All of the patients in this study underwent resting sitting and if ECG has QRS 120 milliseconds, LBBB, LVH, WPW pattern, Paced rhythm, Pericarditis resting ST depression $>1 \mathrm{~mm}$, it will be excluded from the start. The ECG was recorded at a paper speed of $25 \mathrm{~mm} / \mathrm{second}$ at a calibration of $1 \mathrm{mv}$ equals $10 \mathrm{~mm}$. ST segment deviation was measured $60-80$ milliseconds from the $\mathrm{J}$ point, using the TP segment as isoelectric line unless tachycardia caused fusion of the $\mathrm{T}$ and $\mathrm{P}$ waves, in which case the PR segment was used.

\section{Stress ECG (EST): by GE CASE V6.73 machine using Modified Bruce protocol:}

The submaximal treadmill stress test was terminated at least $70 \%$ to $85 \%$ maximal age predicted heart rate as a target end point.

Twelve leads (Mason- Likar lead placement) ECG were monitored continuously and recorded in the sitting position (control) and in the standing position immediately after exercise. The $\mathrm{R}$ wave control measurement was taken in the sitting position for comparison with ST changes.

\section{- ST-segment deviation analysis:}

ST-segment deviation was measured relative to the end of the $P R$ segment because the $\mathrm{T}(\mathrm{U})-\mathrm{P}$ segment during 
exercise was difficult or impossible to measure when HR were fast. Three or more consecutive beats in the same lead with a stable baseline in any lead (other than aVR) compared with the resting trace.

ST depression $>1 \mathrm{~mm}(0.1 \mathrm{mV})$ below the resting level $60-80 \mathrm{msec}$ from the $\mathrm{J}$ point if horizontal or downsloping and > $1.5 \mathrm{~mm}(0.15 \mathrm{mV})$ if upsloping during exercise or immediate recovery period, was defined as positive test (ischemic).

\section{- R wave amplitude (RWA) analysis:}

$\mathrm{R}$ wave amplitude was measured from the isoelectric line to the peak of the $R$ wave in $\mathrm{mm}$ for an average of 6 consecutive beats. The average value was used in the study so the respiratory variation, if any, was minimized. $R$ wave amplitude (RWA) changes were measured in the control (Rest) and in the immediate post exercise period (Immediate Recovery). An increase or no change in $\mathrm{R}$ wave was taken as evidence of an abnormal response, while a decrease in the $\mathrm{R}$ wave was a normal response.

5. Coronary angiogram using the standard Judkins technique from right to left anterior oblique projections with cranial and caudal angulations. The culprit artery was determined from angiographic characteristics of occlusion (occlusion due to thrombus formation or ulceration with decreased contrast density).

\section{Statistical methods:}

The collected data were coded, processed and analyzed using the SPSS (Statistical Package for the Social Sciences) version 22 for Windows ${ }^{\circledR}$ (IBM SPSS Inc, Chicago, IL, USA). Data are expressed as mean \pm standard deviation (SD or number (percent). Comparison between categorical data [number (\%)] in the two studied groups was performed using Chi square test. Comparison between mean values of different variables in the two studied groups was performed using unpaired $t$ test or MannWhitney U test. Receiver operating curve (ROC) was used to calculate the diagnostic indices of EST. P value less than or equal to 0.05 was considered significant. 


\section{RESULTS}

Patients in Group one included 9 males $60 \%$ and 6 females $40 \%$ with a mean age of $47.5 \pm 8.8$. Group two included 27 males $77 \%$ and 8 females $23 \%$ with a mean aged $51.4 \pm 9.6$. There was no statistical difference between both groups as regards the gender and the age distribution of the patients. There were no differences between both groups regarding the presence of hypertension, heart rate, diabetes mellitus, dyslipidemia, family history, body mass index and smoking.

$\triangle \mathrm{RST}$ and RWA were statistically significantly different between the ischemic group and the control group and the changes in ST segment was nonsignificant (Table 1).

Table (1): Demographic data, examination, lipid profile and stress ECG findings regarding studied groups

\begin{tabular}{|c|c|c|c|c|}
\hline \multicolumn{2}{|c|}{$\mathrm{P}_{\text {Parameters }}^{\text {Groups }}$} & $\begin{array}{c}\text { IHD } \\
(n=35) \\
\text { Mean } \pm \text { SD }\end{array}$ & $\begin{array}{c}\text { Control } \\
(n=15) \\
\text { Mean } \pm \text { SD }\end{array}$ & P-value \\
\hline \multicolumn{2}{|c|}{ Age } & $51.4 \pm 9.6$ & $47.5 \pm 8.8$ & $>0.05$ \\
\hline \multirow{2}{*}{ Gender } & Male & $27(77 \%)$ & $9(60 \%)$ & \multirow{2}{*}{$>0.05$} \\
\hline & Female & $8(23 \%)$ & $6(40 \%)$ & \\
\hline \multirow{2}{*}{ Smoking } & Yes & $19(54 \%)$ & $10(66 \%)$ & \multirow{2}{*}{$>0.05$} \\
\hline & No & $16(46 \%)$ & $5(34 \%)$ & \\
\hline \multirow{2}{*}{ FH - IHD } & Yes & $15(43 \%)$ & $9(60 \%)$ & \multirow{2}{*}{$>0.05$} \\
\hline & No & $20(57 \%)$ & $6(40 \%)$ & \\
\hline \multicolumn{2}{|c|}{ MAP } & $91.9 \pm 9.4$ & $92.9 \pm 10.9$ & $>0.05$ \\
\hline \multicolumn{2}{|c|}{ HR } & $79.4 \pm 11.9$ & $80.5 \pm 11.0$ & $>0.05$ \\
\hline \multicolumn{2}{|c|}{ RBS } & $7.5 \pm 1.9$ & $6.5 \pm 1.9$ & $>0.05$ \\
\hline \multicolumn{2}{|c|}{ BMI } & $30.8 \pm 4.0$ & $29.4 \pm 3.7$ & $>0.05$ \\
\hline \multicolumn{2}{|c|}{ LDL } & $5.8 \pm 0.9$ & $3.9 \pm 0.8$ & $<0.001$ \\
\hline \multicolumn{2}{|c|}{ HDL } & $1.0 \pm 0.3$ & $1.1 \pm 0.3$ & $>0.05$ \\
\hline \multicolumn{2}{|c|}{ TGs } & $4.7 \pm 1.3$ & $2.8 \pm 0.6$ & $<0.001$ \\
\hline \multicolumn{2}{|c|}{ RWA Rest } & $10.1 \pm 3.8$ & $11.5 \pm 4.5$ & $>0.05$ \\
\hline \multicolumn{2}{|c|}{ RWA Recovery } & $10.7 \pm 5.2$ & $9.3 \pm 5.5$ & $>0.05$ \\
\hline \multicolumn{2}{|c|}{ RWA Diff } & $0.6 \pm 2.6$ & $-34.2 \pm 2.6$ & 0.007 \\
\hline \multicolumn{2}{|c|}{ ST Seg.Dep } & $1.3 \pm 0.8$ & $0.9 \pm 0.5$ & $>0.05$ \\
\hline \multicolumn{2}{|c|}{$\Delta$ RST } & $1.8 \pm 3.0$ & $-8.3 \pm 2.9$ & 0.001 \\
\hline
\end{tabular}


$\triangle \mathrm{RST}$ was index more useful in detection of coronary artery disease with higher sensitivity and specify among chronic ischemic patients than RWA difference or ST segment depression alone. Particularly when cutoff $\geq-1.65$ cardiac ischemia could be predicted with a sensitivity rate of $98.93 \%$, a specificity rate of $96.00 \%$, a positive predictive value of $96.3 \%$, and a negative predictive value of $98 \%$. RWA at a cut-off point of $\geq-1.2$ was sensitive $95.02 \%$ and specific $94.0 \%$, a positive predictive value of $95.7 \%$, and a negative predictive value of $96.6 \%$. ST segment depression at a cut-off point of $\geq$ 0.45 was sensitive $77.1 \%$ and specific $80 \%$, a positive predictive value of $78.4 \%$, and a negative predictive value of $60.3 \%$ (Table 2).

Table (2): Diagnostic indices of stress ECG using ROC curve in the two studied groups

\begin{tabular}{|c|c|c|c|c|c|c|c|}
\hline & $\underset{Q}{U}$ & 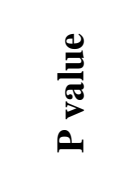 & 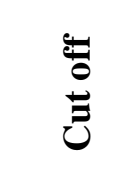 & 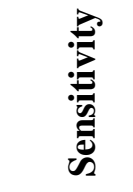 & 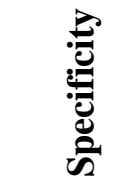 & $\vec{a}$ & $\vec{z}$ \\
\hline RWA Diff & 0.69 & $0.031^{*}$ & $\geq-1.2$ & $95.02 \%$ & $94.0 \%$ & $95.7 \%$ & $96.6 \%$ \\
\hline$\Delta$ RST Index & 0.74 & $0.008^{*}$ & $\geq-1.65$ & $98.93 \%$ & $96.00 \%$ & $96.3 \%$ & $98 \%$ \\
\hline ST Seg Dep & 0.612 & 0.212 & $\geq 0.45$ & $77.1 \%$ & $80 \%$ & $78.4 \%$ & $60.3 \%$ \\
\hline
\end{tabular}

AUC: Area under a Curve, $\mathrm{p}$ value: Probability value

NPV: Negative predictive value, PPV: Positive predictive value

Table (3): Detection of coronary artery disease

\begin{tabular}{|c|c|c|c|c|}
\hline Values & Sensitivity & Specificity & $\begin{array}{c}\text { +ve predictive } \\
\text { value }\end{array}$ & $\begin{array}{c}\text {-ve predictive } \\
\text { value }\end{array}$ \\
\hline$\Delta$ RST $\geq-1.6$ & $98.93 \%$ & $96 \%$ & $96.3 \%$ & $98 \%$ \\
\hline RWA $\geq-1.2$ & $95.02 \%$ & $94 \%$ & $95.7 \%$ & $96.6 \%$ \\
\hline $\begin{array}{c}\text { ST segment } \\
\text { depression }>0.45\end{array}$ & $77.1 \%$ & $80 \%$ & $78.4 \%$ & $60.3 \%$ \\
\hline
\end{tabular}

\section{DISCUSSION}

Insignificant difference of ST segment depression in both groups might be related to the submaximal test. In patients with stable CAD who has done submaximal exercise or cannot exceed $85 \%$ of their target HR. The RWA is a valuable diagnostic test for ischemia than ST segment changes.

Glazier et al. (2013) correlated ST segment changes and ischemia demonstrated by thallium-20 I imaging and showed that the standard ST segment criteria may not correlate as well with ischemia as previously thought in patients with anterior myocardial infarction, the analysis was repeated after patients with anterior myocardial infarction were excluded. This procedure did not change the results significantly.

Deshpande and Birnbaum (2014) demonstrated $\mathrm{R}$ wave changes to be helpful in diagnosing coronary artery disease with exercise testing. $\mathrm{R}$ wave changes were shown to be sensitive independently and, in combination with 
ST segment changes to be superior to ST segment changes alone.

Fifty subjects were enrolled to our study, $72 \%$ were males and $28 \%$ were females with age ranging from above 35 to below 65 years old.

Increases in the $\mathrm{R}$ wave amplitude of the electrocardiogram during exercise or no change have been reported to indicate left ventricular dysfunction or coronary artery obstruction, or both, while decreases have been found consistent with normal left ventricular function. The physiologic mechanism for these changes, once thought to be related to changes in heart volume and the Brody effect, has more recently been suggested to be increased intramyocardial conduction secondary to ischemia, change in contractility or simply changes in heart rate and axis (Deshpande and Birnbaum, 2014).

In our study, when using more complex statistical analysis, $\Delta \mathrm{RST}$ index was more useful in detection of coronary artery disease with higher sensitivity and specify among chronic ischemic patients than RWA difference or ST segment depression alone. Particularly when cutoff $\geq-1.65$ cardiac ischemia could be predicted with a sensitivity rate of $98.93 \%$, a specificity rate of $96.00 \%$, a positive predictive value of $96.3 \%$, and a negative predictive value of $98 \%$.

RWA at a cut-off point of $\geq-1.2$ was sensitive $95.02 \%$ and specific $94.0 \%$, a positive predictive value of $95.7 \%$, and a negative predictive value of $96.6 \%$. ST segment depression at a cut-off point of $\geq$ 0.45 was sensitive $77.1 \%$ and specific $80 \%$, a positive predictive value of $78.4 \%$, and a negative predictive value of $60.3 \%$.
Smith et al. (2012) found similar results but with lower sensitivity of $63 \%$ and a specificity of $79 \%$ for $\mathrm{R}$ wave criteria compared with $48 \%$ and $59 \%$, respectively for $\mathrm{ST}$ segment criteria alone, and reported that an increase or no change in $\mathrm{R}$ wave amplitude in lead CMs immediately after exercise was suggestive of severe coronary artery disease and ischemic left ventricular dysfunction, whereas a decrease in $\mathrm{R}$ wave amplitude was considered a normal response occurring in patients without coronary artery disease.

Harrigan and Brady (2011) clarified the usefulness of R-wave changes during stress testing in the diagnosis of CAD. They studied two groups of patients by means of electrocardiographic (ECG) treadmill testing and coronary arteriography found that R-wave amplitude changes measured from rest to peak exercise are not useful in the diagnosis of coronary artery disease. However, changes in amplitude measured from preexercise to immediately postexercise are a specific and fairly sensitive indicator of coronary artery disease.

Klein et al. (2015) revealed evidence of $\mathrm{CAD}$ in $62.5 \%$ (one vessel disease in $12.5 \%$, two vessel disease in $20 \%$, and three vessel disease in 30\%) of patients. QRS indices of individual lead S of Q, R, and $S$ wave show statistically significant difference in lead II, III, AVF, and V5. Athens score $(\mathrm{aVF}+\mathrm{V} 5) \leq 5 \mathrm{~mm}$ was highly significant and most sensitive (92\%) and specific (80\%) followed by QRS score of leads combination II+III $(<5 \mathrm{~mm})$ being $88 \%$ sensitive and $80 \%$ specific for diagnosis of CAD. 
Comparison of Athens QRS score and coronary angiography revealed that it was $100 \%$ sensitive for detection of two and three vessel disease and only $40 \%$ sensitive for detection of single vessel disease. Alternative QRS score (II+III) showed sensitivity of $100 \%$ for threevessel disease, $87.5 \%$ for two-vessel disease, and $40 \%$ for single vessel disease. They concluded that exercise-induced depolarization abnormality is a useful index not only for diagnosis but also for assessing severity of CAD. However, a larger study especially including those cases with resting depolarization abnormality, e.g., left ventricular hypertrophy (LVH), left bundle branch block (LBBB) might bring out a possible utility of incorporating this parameter in assessing provocable myocardial ischemia when conventional ST-T analysis is not rewarding.

Patel et al. (2010) compared R wave indices of amplitude and ST segment criteria with angiographically determined coronary artery disease. Using $\mathrm{R}$ wave criteria, the sensitivity of exercise testing was $52 \%$ and specificity $63 \%$, compared with 88 and $72 \%$, respectively, using ST segment criteria. No significant correlation was found between the extent of angiographically determined coronary artery disease and $\mathrm{R}$ wave changes.

Smith et al. (2012) demonstrated left ventricular function to be related to $R$ wave amplitude at rest and to its change during exercise. Antzelevitch et al. (2015) did not find $\mathrm{R}$ wave changes during exercise to have a definite physiologic explanation or clinical value.

That cardiac enlargement secondary to congestive heart failure may cause a decrease in $\mathrm{R}$ wave amplitude also contradicts the Brody hypothesis. Correlations of the $\mathrm{R}$ wave to systolic volume and left ventricular ejection fraction suggest an association with contractility. Changes in axis shifts have also been shown to alter $\mathrm{R}$ wave amplitude; this shift of the QRS axis as well as that of the ST segment vector toward the right and posteriorly occur normally during exercise (Barnhill et al., 2012).

\section{CONCLUSION}

$\triangle \mathrm{RST}$ index was more useful in detection of coronary artery disease with higher sensitivity and specify among chronic ischemic patients than RWA difference or ST segment depression alone.

There were variations in age, gender, smoking, family history, hypertension, heart rate, sugar level, BMI and lipid profile between two groups. However, these differences did not reach a statistical significance.

\section{REFERENCES}

1. Antzelevitch C, Yan GX and Viskin S. (2015): Rationale for the use of the terms Jwave syndromes and early repolarization. $\mathbf{J}$ Am Coll Cardiol., 57:1587-1590.

2. Barnhill J, Wikswo J, Dawson AK, Gundersen S, Robertson RM, Robertson D, Virmani $R$ and Smith RF. (2012): The QRS complex during transient myocardial ischemia: studies in patients with variant angina pectoris and in a canine preparation. Circulation, 71:901-911.

3. Deshpande A and Birnbaum Y. (2014): STsegment elevation: Distinguishing ST elevation myocardial infarction from ST elevation secondary to nonischemic etiologies. World J Cardiol., 6:10-13. 
4. Glazier JJ, Chierchia S, Margonato A and Maseri A. (2013): Increase in $S$ wave amplitude during ischemic ST segment depression instable angina pectoris. Am J Cardiol., 59:1295-1299.

5. Greenslade JH, Parsonage $W$ and Ho A. (2015): Utility of routine exercise stress testing among intermediate risk chest pain patients attending an emergency department. Heart Lung Circ., 24(9):879-884.

6. Harrigan RA and Brady WJ. (2011): ECG abnormalities in tricyclic antidepressant ingestion. Am J Emerg Med., 17:387-393.

7. Klein LR, Shroff GR and Beeman W. (2015): Electrocardiographic criteria to differentiate acute anterior ST-elevation myocardial infarction from left ventricular aneurysm. Am J Emerg Med., 33:786-790.

8. Macintyre CR, Heywood AE and Kovoor P. (2013): Ischaemic heart disease, influenza and influenza vaccination: a prospective case control study. Heart, 99(24):1843-8.

9. Mehta PK, Wei J and Wenger NK (2015): Ischemic heart disease in women: a focus on risk factors. Trends in Cardiovascular Medicine, 25 (2): 140-51.

10. Mittleman MA, Maclure $M$ and Tofler GH. (2011): Triggering of acute myocardial infarction by heavy physical exertion: protection against triggering by regular exertion: Determinants of Myocardial
Infarction Onset Study Investigators. N Engl J Med., 2329: 1677-1683.

11. O'Gara PT, Kushner FG and Ascheim DD. (2013): American College of Cardiology Foundation/American Heart Association Task Force on Practice Guidelines. 2013 ACCF/AHA guideline for the management of ST-elevation myocardial infarction: a report of the American College of Cardiology Foundation/American Heart Association Task Force on Practice Guidelines. Circulation, 127 (4): 362-425.

12. Patel MR, Peterson ED, Dia D, Brennan JM, Redberg RF, Anderson HV, Brindis RG and Douglas PS. (2010): Low diagnostic yield of elective coronary angiography. $\mathrm{N}$ Engl J Med., 362:886-895.

13. Skyler JS, Bakris GL and Bonifacio E. (2017): Differentiation of diabetes by pathophysiology, natural history, and prognosis. Diabetes, 66:241-255.

14. Smith SW, Dodd KW and Henry TD. (2012): Diagnosis of ST-elevation myocardial infarction in the presence of left bundle branch block with the ST-elevation to $\mathrm{S}$-wave ratio in a modified Sgarbossa rule. Ann Emerg Med., 60:766-776. 
تقيم إرتفاع موجة أر مقارنة بانخفاض قطعة اس-تى باستخدام

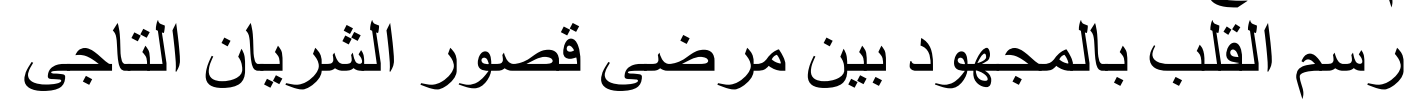

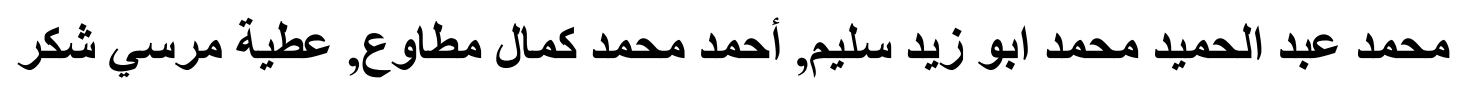
قسم القلب والأوعية الاموية, كلية الطب، جامعة الأزهر

E-mail: muhammadabouzeid87@gmail.com

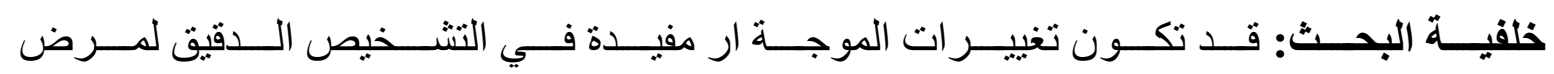

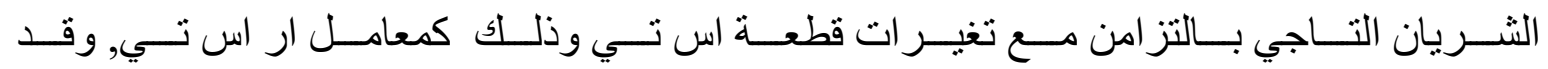

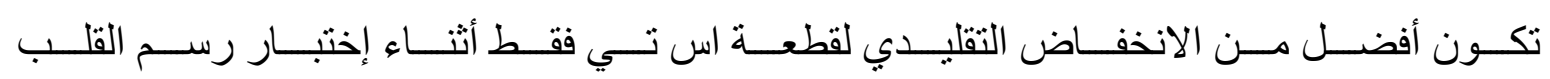
بالمجهود

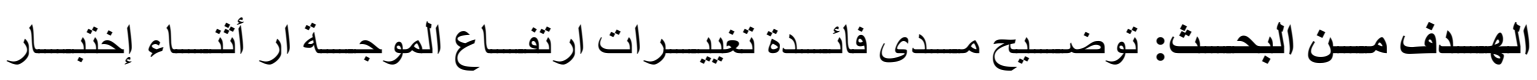
رسم القلب بالمجهود في زيادة دقة تشخيص قصور الثريان التاجى.

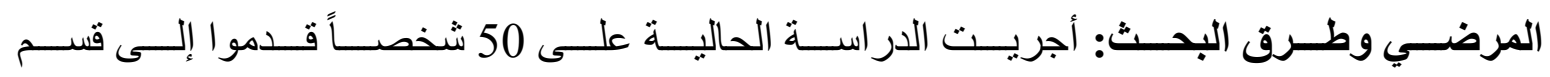

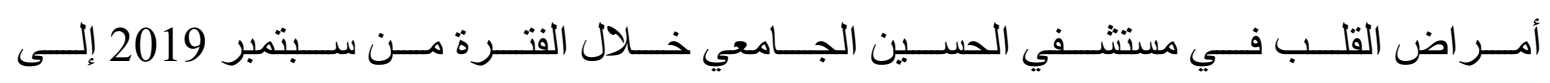

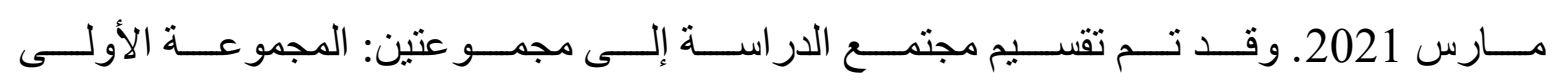

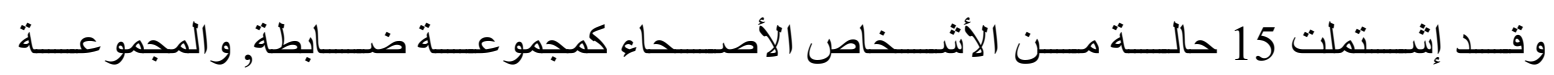

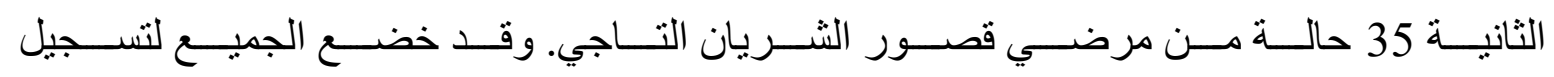

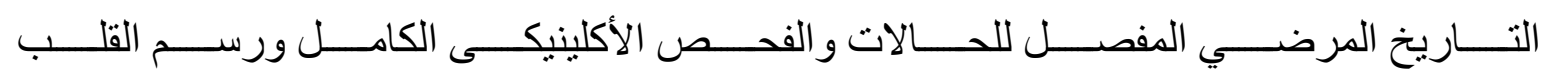
العادي وبالمجهود وقسطرة الثر ايين التاجية.

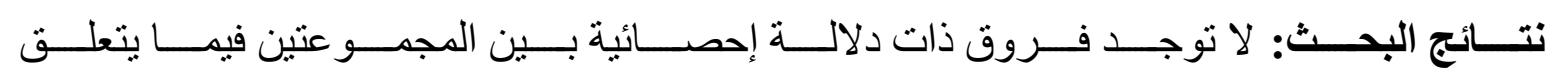

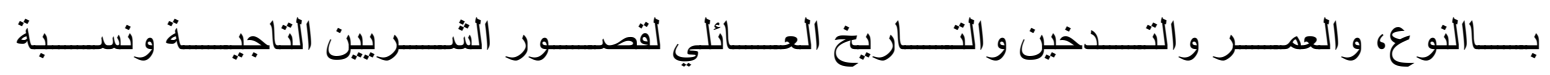

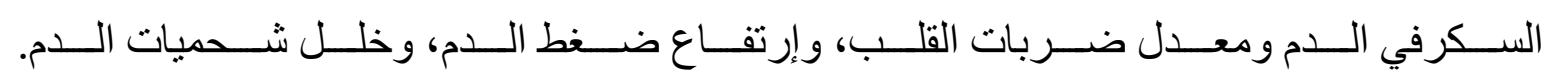

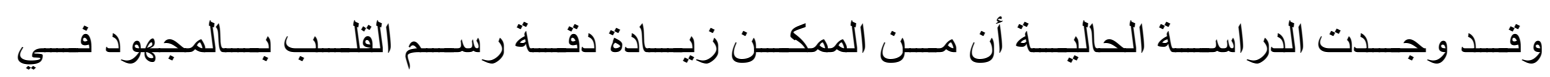

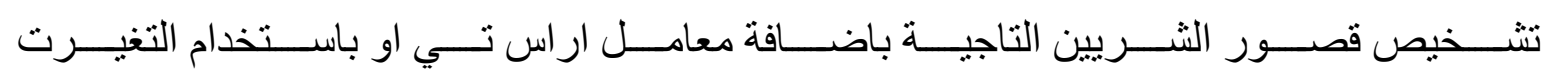
في موجة ار مقابل الطريقة التقليدية باستخدام انخفاض قطعة اس تي فقط. 


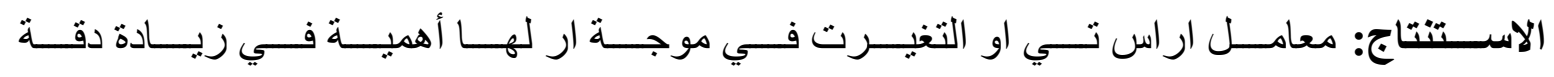

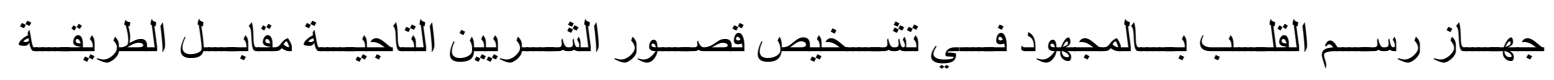
التقليدية بإستخدام إنخفاض قطعة اس تي فقط.

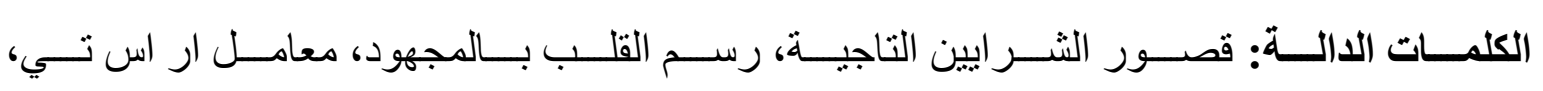
التغير ات في موجة ار. 